DOI: $10.21767 / 2171-6625.100009$

\section{Insular Cortex and Insular Epilepsy}

\section{Abstract}

Insular cortex epilepsy is an under-recognized, localization-related syndrome. The presence of this condition could account for the surgery treatment failure seen in a portion of patients with temporal epilepsy, as well as a portion of those with frontal and parietal lobe epilepsy. Most cases of intractable temporal epilepsy are associated with insular cortex involvement. In this review, we will discuss the associations of insular cortex with other nervous cortex systems anatomically and functionally in order to correctly understand the insular lobe; the relationship between insular epilepsy and temporal epilepsy, the independence of insular epilepsy, and differences of insular epilepsy between the independent epileptogenesis and epileptic system as well as the concept of epileptic foci-complex were also discussed based on the current literature and our clinical experience in the treatment of epilepsy.

Keywords: Insular cortex; Epilepsy; Temporal epilepsy; Intractable epilepsy

Received: April 29, 2015; Accepted: June 15, 2015; Published: June 18, 2015

\section{Introduction}

The concept of "insular cortex epilepsy" was first proposed in the 1950s with the observation that the epileptic symptoms resulting from the stimulation of the insular cortex were similar to those of temporal epilepsy to the extent that it was difficult distinguish between them [1-3]. In subsequent years, clinical data have demonstrated that in patients treated with surgery, approximately $70 \%$ experience satisfactory benefits, almost $20 \%$ experience suboptimal benefits, and $10 \%$ experience no benefit at all. In these latter patients, classified as having intractable epilepsy, the main reason for treatment failure may be insular cortex involvement, as simple temporal lobectomy did not resolve epileptic symptoms [4-6].

The insular lobe belongs to the limbic system and is regarded as the fifth lobe of the brain. This lobe is closely associated with visceral activities and the emotion area and abuts the motor, feeling, and language areas. The insular cortex possesses a variety of functions, such as memory, drive, and emotion, as well as higher autonomic control of gustation and olfactory sensation [7-10]. Tumors in the insular lobe, if left untreated, could induce dysfunction in multiple systems and jeopardize life [11-15]. The onset of insular cortex epilepsy is difficult to record by scalp electrode because of its deep anatomic location and is limited by lateral clustered middle cerebral artery. Therefore, routine scalp electroencephalograph (EEG), which is considered the

\section{Tao Sun, Feng Wang and Jianqi Cui*}

\begin{abstract}
Ningxia Key Laboratory of Cerebrocranial Diseases, The Incubation Base of National Key Laboratory, Ningxia Medical University, Yinchuan, Ningxia Hui Autonomous Region PR China, 750004
\end{abstract}

\section{Corresponding author: Jianqi Cui \\ $\equiv$ jqcui@nxmu.edu.cn \\ Ningxia Key Laboratory of Cerebrocranial Diseases, Ningxia Medical University, 1160 Shengli Street, Yinchuan, Ningxia, 750004, PR China.}

Tel: 15296900235

Fax: 0951-6880697

Citation: Tao Sun, Feng Wang. Insular Cortex and Insular Epilepsy. J Neurol Neurosci. 2016, 6:1.

most useful and valuable approach in epilepsy diagnosis, plays a less important role in insular epilepsy. Because it is difficult to ascertain whether the epilepsy originated from the mesial temporal lobe and spread to the insular lobe or vice versa, the existence of insular epilepsy remains under debate [16-

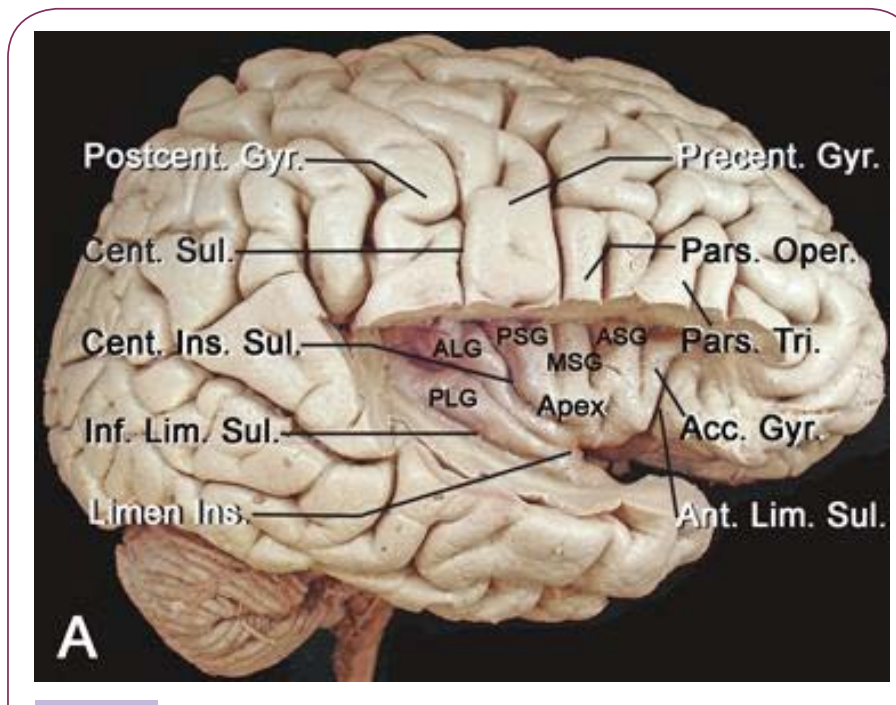

Figure 1 Anatomy of insular cortex [4]. 
18]. With improved investigative techniques, such as the miniaturized electrode in stereo-EEG and advances in the orientation of magnetic resonance imaging [MRI], the research in this field has received increased attention $[19,20]$.

\section{Is the Insular Lobe a Real Island?}

Vicq first studied the insular lobe in 1786 and described it as "the circle round located between lateral fissure and corpus striatum". The concept of the insular lobe was formally proposed by the German physician and anatomist Dr. Johann Christian Reil in 1809 and was named after his name as the Island of Reil [1-3]. The insula is a highly developed structure, is entirely encased within the brain at the depth of the Sylvian fissure and is covered by the frontal, parietal and temporal opercula. Clark examined the anatomic position of the insular lobe in 1896 and was the first to describe it as "the fifth lobe of the brain" [3]. The knowledge of insular functions is primarily derived from its anatomic position and cellular composition. It was considered to be an isolated lobe inside the human brain and belong to the autonomic nervous system that is primarily involved with visceral sensation (including pain), movement (including gustation), and emotions [7-10].

The current research in anatomy provides persuasive evidence that the insula has wide connections with the neocortex, basal ganglia, thalamus, limbic structures and olfactory cortex [21,22,61-64]. These numerous connections underlie the complex functional spectrum of the insular lobe (Figure 1). The white matter tracts study with Klinger techniques and MRI-DTT (Magnetic Resonance Imaging-Diffusion Tensor Tractography) demonstrated that the insular lobe possesses complicated structures and is closely associated with surrounding lobes, as well as the limbic system and paralimbic system through white matter fibers tracts, which disproves the theory that the insular lobe is an isolated island $[22,23]$. These wide connections with surrounding structures suggest that the insular lobe also possesses multiple functions, which include somatosensory, auditory, and language, as well as having a primary visceral/autonomic sensory, motor, and a supplementary motor area [24,25].

From an embryology perspective, the insular lobe is the structure between the neocortex and paleopallium with a triangular shape that is surrounded by the anterior, superior and mesial limiting sulci; the inverted pyramidal shape has insular apex and is closed by the Sylvian fissure. The fronto-orbital operculum, frontoparietal operculum and temporal operculum are distinguished with these sulci. When the insular operculum is opened, the insular lobe is divided into the anterior insula and posterior insula by the central insular sulcus. The insular lobe is also divided into three parts according to its cortical structure, namely, the agranular cortex in the center, surrounded by the dysgranular cortex, with the granular cortex being outermost; the granular cortex extends to the caudal border of the Island of Reil [26]. Anatomic studies have completed the demarcation of the insular lobe [4] and identified anatomic markers, including an important index point, the posterior-inferior point (Figure 2). The use of improved DTT techniques in the tempo-insular area has enabled easier recognition and localization to determine the anatomic landmark of temporal stem; the anterior limit of the temporal stem is the limen insula and the posterior limit is the posteroinferior insular point [4]. These distinct anatomic definitions further our understanding of the relationship between the insular lobe and its surrounding structures [27] (Figures 2 and 3).

The relationship between the insular lobe, the mesial temporal lobe and the cortex of insular opercula, as well as the connections of whiter matter tracts between these areas, warrants further investigation.

The function of the insular lobe and its role in the central nervous system has been heavily investigated [7-12]. Thinking and feeling have been demonstrated to be integrated in the insular lobe cortex $[28,29]$. The physiological signals received by the body are controlled by the insular lobe cortex, producing subjective feeling to instruct the body to respond appropriately to maintain homeostasis. The insular lobe cortex receives signals from sensory receptors distributed in the skin and internal organs, perceiving such sensations as cold, hot, itch, pain, smell, thirst, muscular soreness, visceral sensitivity and dyspnea. The signals from the insular lobe cortex transfer to other related brain structures, particularly the anterior cingulate cortex and anterior frontal cortex, where a final decision is made on the basis of sensory information [30].

The insular lobe cortex also functions to reflect the body's status. Information concerning the status of tissues and organs are received by special sensory receptors and travel via different pathways through the spinal cord to the brain stem and higher structures, such as the insular lobe cortex or brain cortex. The information is processed into an action such that the body will respond appropriately to stimulation. The insular lobe cortex monitors the body's needs and helps to enable activities to satisfy these needs. The insular lobe cortex is also important in making essential responses. For example, when individuals decide to go out in cold weather, the body has already prepared itself to cope with the cold by

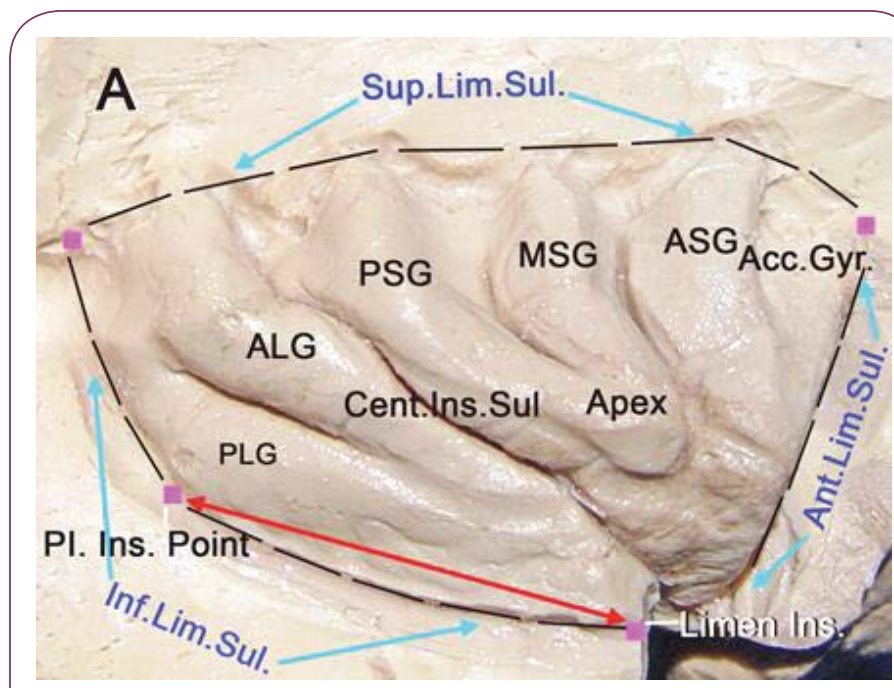

Figure 2 Demarcation of insular cortex [4]. 


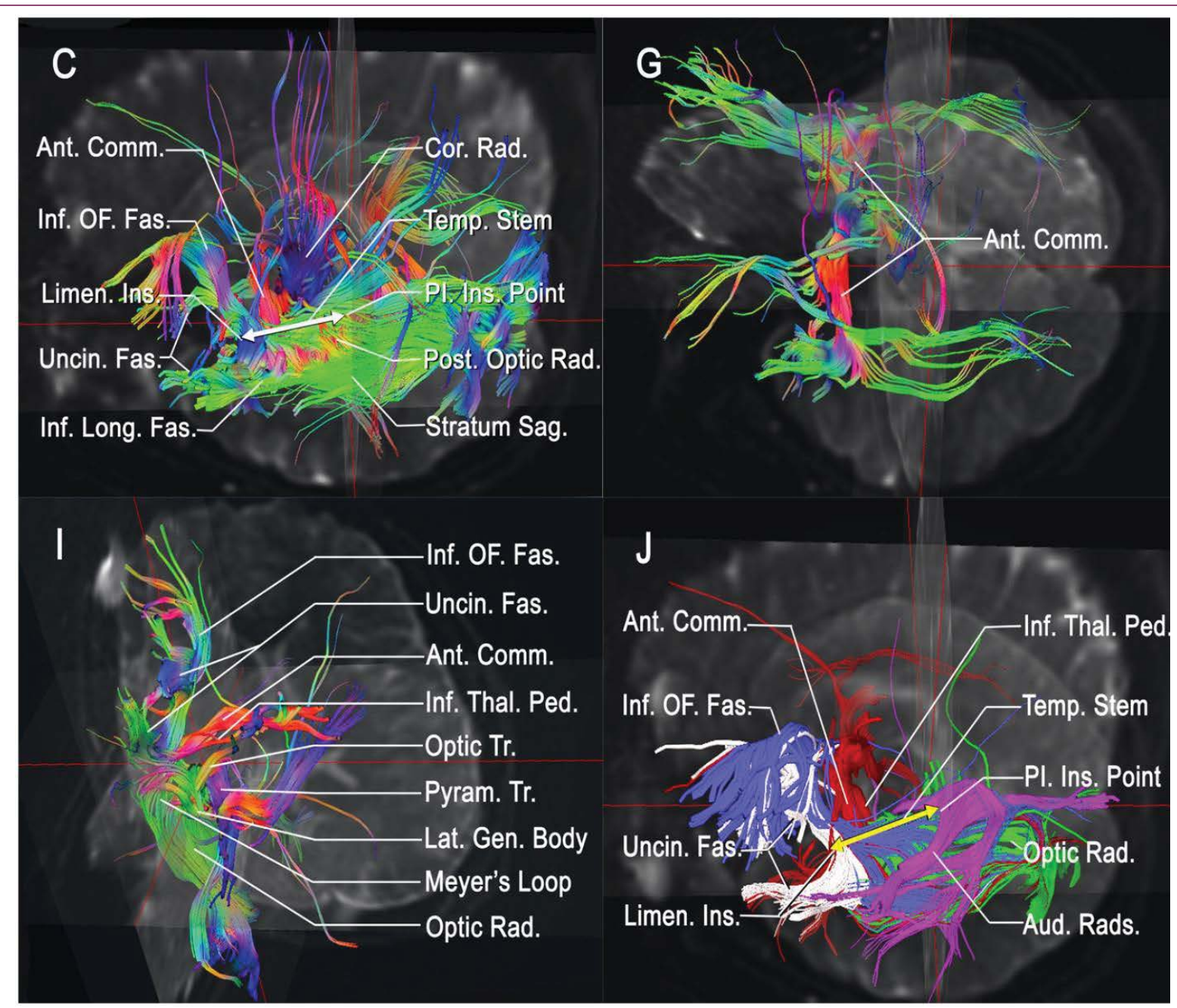

Figure 3 DTT study of temporal stem and insular cortex [4].

rearranging blood distribution adjusting metabolism.

It is not difficult to see that the insular lobe cortex has wide connections with surrounding brain structures and is involved in many important activities of the central nervous system. Clearly, the insular lobe cortex is not simply an isolated island, and its roles and functions warrant further research.

\section{Relationship between Insular Epilepsy and Temporal Epilepsy}

The concept of "insular cortex epilepsy" was first proposed by Guillaume [16-18] and Mazars [31]; subsequently, it was reported by Penfield [24,25] and Jasper [32] that seizures emanating from the insula may have semiology similar to that of temporal lobe seizures. About half of the patients with temporal epilepsy had intermittent discharge in the insular lobe cortex during surgical treatment under local anesthesia, as detected by intraoperative electrocorticography and applied electric stimuli. Stimulation of the insular lobe cortex also induced symptoms similar to those observed during routine seizure onset. These discoveries demonstrate that the insular lobe cortex may also possess the epileptogenic potency, and insular epilepsy may be intermingled with temporal epilepsy [33]. These investigators proposed that persistent seizures following a temporal lobe resection may be explained by failure to remove an epileptogenic focus within the insula and prompted resections of the temporal lobe and portions of the insula in patients with robust interictal insular spikes during intraoperative electrocorticography. However, the use of this surgical strategy declined following the report by Silfvenius [34] that insular resection, when added to temporal lobectomy, failed to increase epileptic control and significantly increased surgical morbidity.

The key point to identifying epileptogenic foci is dependent on the EEG. However, interictal or ictal surface EEG studies are not very helpful in residing in the insula, because of the hidden, distant location of insular lobe cortex with relation to scalp electrodes and is spread rapidly such that distinguishing whether the symptoms originate from the insular or its surrounding structures is often not possible. Isnard et al. $[35,36]$. directly stimulated the insular lobe of the patients and observed the induced symptoms, and concluded that several common characteristics of seizures appeared in individuals with insular epilepsy: 1, consciousness; 2 , a sensory premonitory symptom before the paroxysm, which always manifested as a sensation of electric current or burning heat restricted in the perioral or larger area (i.e., face-shoulderhand and trunk, upper limbs-body-lower limbs); 3, an ever-present 
abnormal sensation of retrosternal pain, abdominal elongation and distension, nausea and vomiting, dyspnea, mogiphonia or anarthria; 4, pharyngeal symptoms of movement and sensation of paroxysm, accompanied with contralateral hands of the discharge side, and extending to the cervix, to grab and scratch; 5, movement symptoms in the ipsilateral or contralateral discharge side, such as facial or upper limb spasm, rotating head or eyes, and systemic dystonia.

As stated above, insular epilepsy has similar symptoms to those of temporal epilepsy. In most patients, primary insular epilepsy is difficult to distinguish from temporal epilepsy, and the two are usually confused and intermingled. In the recently reignited interest on insular research with chronic depth EEG electrodes to examine the role of the insular cortex in patients with possible temporal lobe epilepsy (TLE), Isnard et al. determined that of 21 patients with drug-refractory TLE, two patients had seizures originating from the insular lobe. Neither patient achieved seizure control following a temporal resection that spared the insular cortex, which was in contrast to excellent seizure outcome in 15 of 17 patients who did not have insular onset seizures. Aghakhani et al. [39]. reported that in six patients with electroclinical findings suggestive of TLE, there were somatosensory auras suggestive of extratemporal ictal onset. None of these patients experienced improvement after temporal lobe surgery, despite intracranial EEG evidence that the temporal lobe was involved in generating seizures. Another region where the ictal onset of seizures could not be controlled by removal of the epileptic focus is the inferior parietal lobe cortex; it has been suggested that the seizures occurring in this area may also have insular lobe involvement. This speculation is difficult to confirm because of a lack of intracerebral electrodes placed directly within the insula. Ryvlin et al. [40-42] reported that three patients with drugresistant, nonlesional nocturnal frontal lobe epilepsy showed ictal onset primarily involving the insular lobe, rather than the mesial or orbitofrontal cortex, when monitored with intracerebral EEG. Taken together, these findings support the original concept that a lack of recognition of insular epilepsy could account for the failure of surgical treatment in patients with temporal, as well as parietal or frontal, epilepsy. Approximately $20 \%$ of patients with temporal epilepsy experience failure with surgical therapy; we believe the reason is insular lobe cortex involvement and that the epileptogenic foci may have originated from the insula. If insular epilepsy can be distinguished from temporal epilepsy, insular epilepsy could be better treated with surgical resection of the insular epileptogenic foci with further improvements in the efficacy of temporal epilepsy treatment with the resection of hippocampus sclerosis combined with stereotaxic lesion or resection of the insular epileptic foci [43-50].

\section{The Insular Lobe is where Temporal Symptoms Occur}

The symptoms of independent insular epileptic seizure are observed in the clinic. Our institute has observed 18 cases of insular epilepsy during October 2008 to October 2010 [51]. In these patients, there were 7 males and 11 females, aged from 6 to 54 (average 35 years' old), typical symptoms include throat discomfort, chest constriction, dyspnea, unpleasant abnormal somatesthesia, pyrexia or pain originating from around the mouth that may spread broadly to other parts of the body. Other symptoms include mogilalia and abnormal somatic motor performance. These clinical manifestations suggest the involvement of a cortex connected with the insula in the originating and spreading of the insular focal epilepsy. Isnard et al. reported five cases of insular cortex epilepsy by stereo electroencephalogram (SEEG) recordings. These researchers determined that the patients with atypical TLE, as suggested by the presence of ictal symptoms compatible with early spread of seizures to the suprasulvian opercular cortex (lip and face paresthesia, laryngeal constriction, tonic or clonic movements of the face, dysarthria or motor aphasia, gustatory illusions, hypersalivation, postictal facial paresis) nor to the infrasylvian opercular cortex (auditory hallucinations or early sensory aphasia).

The insular lobe has been considered as the fifth lobe of the brain. To date, the model of insular electrical kindling has been successfully established [51,52]. Insular kindling is much faster than amygdala kindling $[53,54]$. Under some pathophysiological conditions, such as insular cavernous hemangioma and lowgrade gliomas, operculum lesions may provoke the insular cortex, producing the location-specific insular epileptic seizures. This type of epilepsy could be treated by excision of the insular epileptic foci $[33,51,59]$.

When the insular cortex is partially involved and/or is a single epileptogenic focus, and not only as a relay structure in seizure propagation, the concept of "insular epilepsy" is logical. As for any other types of partial seizures, the concept of a distributed epileptogenic network can be applied to describe insular seizures, the insular lobe also acts as the node of epileptogenic networks, in which seizures could spread to other locations by three different ways, and the spread networks would also be mutual and connected with each other; further investigations in this topic is warranted.

With increasing awareness of the various aspects of insular cortex epilepsy, indications for insular cortex sampling with intracerebral electrodes have been expanded. Nguyen et al. [5] reported that the decision to sample the insula with intracerebral electrodes is made in the cortex of (a) non-lesional parietal lobe (PL)-like epilepsy, (b) non-lesional frontal lobe-like epilepsy, (c) nonlesional temporal lobe-like epilepsy, and (d) atypical temporal lobe-like epilepsy, as defined by the Lyon group. Aided by intracerebral electrodes, these researchers identified four cases of insular seizures in 18 consecutive patients [56]. Therefore, insular cortex epilepsy may not be rare in patients with refractory epilepsy who are surgical candidates.

\section{The Insular Lobe is a Part of the Epileptogenetic System: The Concept of an "Epileptic Foci Complex"}

In terms of anatomy, the insular cortex is a part of the cerebral cortex network. As previously discussed, it has broad connections with other cortex systems. Although the insular lobe has distinct anatomic demarcations, such as Limiting Sulcus, it also possesses transitional cell structure definitions connected with circumjacent 
cortex and forms intensive internuncial structure. Therefore, the function of the insula lobe and the roles that it plays in epileptic seizures is much more complicated; therefore, it should no longer be considered as an isolated functional center; the name "insula" is confusing when its actual functions are considered. Augustine et al. [7] has described the connection systems of the insular lobe, stating that the insular cortex is connected with the surrounding cortex such as amygdala, nuclei basales, and all of the cerebral cortex except the occipital lobe. In the current established structure systems, such as the lateral fissure-insula, temporal-limbic-insula and interior orbitofrontal lobe-insula, increasing attention has focused on the insular lobe cortex, as the epileptic seizure symptoms caused by epileptic discharges from each area would ultimately be attributed to insular lesions. Indeed, these were only partial symptoms of all the insular epileptic manifestations. These broader structural connections of the insular cortex with the cerebral cortex could explain why it is difficult to distinguish pure insular epilepsy from all the epileptic seizures where the epileptogenic zones are located beyond the insular lobe cortex.

As we discussed above, the insular lobe cortex is the part of the cortex network distributions. There might be three main propagation pathways in insular epileptogenic network: the first pathway is the temporo-perisylvian-insular networks that include the various brain regions bordering the sylvian fissure, i.e., the frontal, parietal and temporal operculum, together with the insula; the second pathway is the temporo-limbic-insular networks, which primarily involve the mesial temporal structures and/or the temporal pole; the final pathway is the Mesial and orbito frontal-insular networks, in the context of the so-called nocturnal frontal lobe epilepsy.

The insular lobe forms the "epileptic foci complex", along with the hippocampus and amygdala, with the latter acting as the transitional effector [37,54]. Pathological studies have demonstrated that after insular kindling, synaptic reconstruction and mossy fiber sprouting appeared in the hippocampus area, with electrophysiological long-term potentiation [57]. These manifestations suggest changes in synaptic plasticity and support an important spreading mechanism in the insular epileptic system. From a clinical point of view, there are still many issues to be clarified. In the case of "insular epilepsy", should we remove the local epileptic foci in the insular, or include the hippocampus and amygdala as well? If an epileptogenic zone associated with the insula exists, a simple resection of the insular lobe would not solve the problem, and the epilepsy would still persist. What are the conditions of an effective resection of the insular epileptic foci?

The insular lobe does not exist solely with the amygdalahippocampus complex, and it is not only part of the propagation pathway of temporal epilepsy, as there are other propagation pathways. The insula is a part of epileptogenic networks, including frontal epilepsy characterized with nocturnal hyperkinesia. The insular lobe is the primary silence zone of clinical onset. In general, when the insular cortex is considered to be partially involved with and/or is a transit station in the epileptic spreading pathway, and not just as a simple epileptic foci, insular epilepsy exists as an entity in epileptogenic networks.

\section{The Internal Relationship between "Independent Epileptogenesis" and Epileptic System}

As we discussed above, in most situations, insular is a part of the epileptogenic network and is also involved in the propagation pathways or act as a spreading station for the epileptogenic focus of another brain areas $[21,33,56]$. There are unanswered questions, such as what is the condition for an independent insular epilepsy? How can we distinguish between the epilepsies originating from the insula with those from another cortex? What is the relationship between the hippocampal circuit of the temporal epilepsy and the circuit of insular epilepsy? What are the associations and interactions among the insular epileptogenic networks?

To answer these questions, further investigations with precise and intensive study are needed. Our institute has focused on insular epilepsy for several years, and we have conducted a series of comprehensive studies that cover different categories $[14,22,23]$. In a general anatomic study of white matter tracts using the Klinger technique and neuroimaging study using MRDTT technique, we found that the insular lobe has complicated structures, and despite its name, is actually not an "island". Its white matter fibers connect widely with surrounding lobes, the limbic system and paralimbic system [22,23,27,53].

The insular lobe serves as a component of the network structure of cortex distribution. There are three spreading pathways in the epileptic system: (1) temporal-lateral fissure-insular lobe, mainly the insular operculum; (2) temporal-limbic systeminsular lobe, inner structure of tempus and/or temporal pole; (3) inner side-orbitofrontal-insular lobe, including the inner side of the insular lobe and the orbitofrontal cortex.

It has been demonstrated that the electric stimulation kindling model is the best animal model for studying the mechanism of temporal epilepsy. We have established a stable, reliable insular electric kindling model to evaluate insular epilepsy, and utilized the methods of electrophysiology, recognition, pathology, and molecular biology to demonstrate that the insular lobe, as with the frontal, temporal, parietal and occipital lobes, can be an independent focus for epilepsy and is not just a spreading component of the temporal epileptic pathway [37,38,52-55].

In a rat insular kindling model, the changes in the time interval of reactive synaptic plasticity occurred in the hippocampus of the inner side of the temporal lobe, which further confirms that the outbreak and maintenance of insular epilepsy is closely associated with the hippocampus $[23,57,58]$. In the complicated insular epileptogenic networks, the insular lobe, together with the hippocampus and amygdaloidal nucleus, make up the "focus complex" and act as a relay-operated effector in epilepsy propagation.

Induction of rat insular electric kindling model causes mossy fiber 
sprouting inside the hippocampus, prolongs the time interval and alter the expression of certain proteins associated with multiple reactive synaptic plasticity; these comprise the mechanisms of insular epileptic outburst $[57,59,60]$.

When the insular lobe is considered to be an essential component or as a simple epileptic focus (but not as a transit structure in the epileptic propagation), this characterization establishes the concept of "insular epilepsy".

According to these findings, we propose the concept of an "insular epilepsy" and that the insular lobe has original (or independent) and relay-operated (or compound) functions.

\section{Conclusions}

The insular lobe is not an isolated "island", as it has broad anatomic and functional connections with other important brain structures. Insular epilepsy is closely associated with temporal epilepsy, which may account for suboptimal treatment outcomes in temporal epilepsy, as the insular lobe is involved, and the temporal lobe is not the sole target for treatment. The insular lobe could be an independent zone in which epileptic seizures take place. The insular lobe is a part of the epileptogenic networks, and it forms an epileptogenic foci complex with the temporal and orbitofrontal lobes, as well as the hippocampus and amygdala. The relationship between the independent epileptogenesis of the insular lobe and the epileptogenic networks is close, being characterized by broad internal connections.

\section{Acknowledgments}

This work was supported by a Chinese National scientific foundation grant to Dr. Jianqi Cui (81260197) and the Chinese National 973 project grants to Dr. Tao Sun (2012CB722408) and Dr. Jianguo Niu( 2014CB560711). The authors thank Dr. Zheng Liu in the Department of Neurosurgery of the General Hospital affiliated to Ningxia Medical University for the clinical data collections and literature support. 


\section{References}

1 Reil JC (1809) Die sylvische Grube. Arch Physiol (Halle) 9: 195-208.

2 Reil JC (1809) Untersuchungen uber den Bau des grossen Girhirns im Mensche. Vierte Fortsesung VIII. Arch Physiol (Halle) 9: 136-146.

3 Clark TE (1896) The comparative anatomy of the insula. J Comp. Neurol 6: 59-100.

4 Sun T, Wang F, Cui J (2013) Insular Epilepsy (1stedn), People's Medical Publishing House, Beijing.

5 Nguyen DK, Nguyen DB, Malak R, Bouthillier A (2009) Insular cortex epilepsy: an overview. Can J Neurol Sci 36 Suppl 2: S58-62.

6 Nguyen DK, Nguyen DB, Malak R, Leroux JM, Carmant L, et al. (2009) Revisiting the role of the insula in refractory partial epilepsy. Epilepsia 50: 510-520.

7 Augustine JR (1996) Circuitry and functional aspects of the insular lobe in primates including humans. Brain Res Brain Res Rev 22: 229-244.

8 Ostrowsky K, Isnard J, Ryvlin P, Guénot M, Fischer C, et al. (2000) Functional mapping of the insular cortex: clinical implication in temporal lobe epilepsy. Epilepsia 41: 681-686.

9 Shelley BP, Trimble MR (2004) The insular lobe of Reil--its anatamico-functional, behavioural and neuropsychiatric attributes in humans--a review. World J Biol Psychiatry 5: 176-200.

10 Cascino GD, Karnes WE (1990) Gustatory and second sensory seizures associated with lesions in the insular cortex seen on magnetic resonance imaging. J Epilepsy 3: 185-187.

11 Duffau H, Capelle L (2005) Incontinence after brain glioma surgery: new insights into the cortical control of micturition and continence. Case report. J Neurosurg 102: 148-151.

12 Ostrowsky K, Isnard J, Ryvlin P, Guénot M, Fischer C, et al. (2000) Functional mapping of the insular cortex: clinical implication in temporal lobe epilepsy. Epilepsia 41: 681-686.

13 Ostrowsky K, Magnin M, Ryvlin P, Isnard J, Guénot M, et al. (2002) Representation of pain and somatic sensation in the human insula: a study of responses to direct electrical cortical stimulation. Cerebral Cortex 12: 376-385.

14 Wang F, Xu Y, Li Z, Xu J, Liu Z, et al. (2012) Surgical strategies and seizure outcomes of patients with epilepsy due to insular lesions. Chin J Neurosurg 28: 392-395.

15 Kerkhof M, Vecht CJ (2013) Seizure characteristics and prognostic factors of gliomas. Epilepsia 54 Suppl 9: 12-17.

16 Guillaume MMJ, Mazars G. Cinq (1949) CAS de foyers épileptogènes insulaires opérés. Soc Française de Neurol 766-769.

17 Guillaume MMJ, Mazars G (1949) Technique de résection de l'insula dans les épilepsies insulaires. Rev Neurol 81: 900-903.

18 Guillaume J, Mazars G, Mazars Y (1953) [Surgical indications in socalled temporal epilepsy]. Rev Neurol (Paris) 88: 461-501.

19 DeSalvo MN, Douw L, Tanaka N, Reinsberger C, Stufflebeam SM (2014) Altered structural connectome in temporal lobe epilepsy. Radiology 270: 842-848.

20 Blauwblomme T, David O, Minotti L, Job AS, Chassagnon S, et al. (2013) Prognostic value of insular lobe involvement in temporal lobe epilepsy: a stereoelectroencephalographic study. Epilepsia 54: 1658-1667.
21 Chevrier MC, Bard C, Guilbert F, Nguyen DK (2013) Structural abnormalities in patients with insular/peri-insular epilepsy: spectrum, frequency, and pharmacoresistance. AJNR Am J Neuroradiol 34: 2152-2156.

22 Wang F, Sun T, Li X, Xia H, Li Z (2011) Microsurgical and tractographic anatomical study of insular and transsylvian transinsular approach. Neurol Sci 32: 865-874.

23 Wang F, Sun T, Li XG, Liu NJ (2008) Diffusion tensor tractography of the temporal stem on the inferior limiting sulcus. J Neurosurg 108: $775-781$.

24 Penfield W, Faulk ME Jr (1955) The insula; further observations on its function. Brain 78: 445-470.

25 Penfield W, Jasper HH (1954) Epilepsy and the functional anatomy of the human brain. Boston, Little, Brown.

26 Molnár Z, Kaas JH, de Carlos JA, Hevner RF, Lein E, et al. (2014) Evolution and development of the mammalian cerebral cortex. Brain Behav Evol 83: 126-139.

27 Xu J, Wang F, Miao L, Wang EJ, Shen FZ, et al. (2012) The study of the microsurgical anatomy on the relationship of the claustrum with its adjancent white matter fiber tracts. Chin J Neurosurg 28: 513-517.

28 Singer T, Critchley HD, Preuschoff K (2009) A common role of insula in feelings, empathy and uncertainty. Trends Cogn Sci 13: 334-340.

29 Singer T (2006) The neuronal basis and ontogeny of empathy and mind reading: review of literature and implications for future research. Neurosci Biobehav Rev 30: 855-863.

30 Craig AD (2002) How do you feel? Interoception: the sense of the physiological condition of the body. Nat Rev Neurosci 655-666.

31 Mazars G (1970) Criteria for identifying cingulate epilepsies. Epilepsia 11: 41-47.

32 Magiorkinis E, Diamantis A, Sidiropoulou K, Panteliadis C (2014) Highights in the history of epilepsy: the last 200 years. Epilepsy Res Treat 2014: 582039.

33 Desai A, Bekelis K, Darcey TM, Roberts DW (2012) Surgical techniques for investigating the role of the insula in epilepsy: a review. Neurosurg Focus 32: E6.

34 Silfvenius H, Gloor P, Rasmussen T (1964) Evaluation of insular ablation in surgical treatment of temporal lobe epilepsy. Epilepsia 5: $307-320$.

35 Isnard J, Guénot M, Ostrowsky K, Sindou M, Mauguière F (2000) The role of the insular cortex in temporal lobe epilepsy. Ann Neurol 48: 614-623.

36 Isnard J, Guénot $M$, Sindou $M$, Mauguière $F$ (2004) Clinical manifestations of insular lobe seizures: a stereoelectroencephalographic study. Epilepsia 45: 1079-1090.

37 Zhang X, Wang F, Miao L, Song ZM, Wang EJ, et al. (2013) Role of insular cortex in amygdala-kindled seizures in rats. Chin J Neurosurg 12: 119-122.

38 Wang CS, Fun T, Wang F, Bai J (2007) The establishment of SpragueDawley rat models with insular kindling chronic epilepsy induced by electrical stimulation. Chin J Neuromed 6: 925-927.

39 Aghakhani Y, Rosati A, Dubeau F, Olivier A, Andermann F (2004) Patients with temporoparietal ictal symptoms and inferomesial EEG do not benefit from anterior temporal resection. Epilepsia 45: $230-236$. 
40 Ryvlin P, Minotti L, Demarquay G, Hirsch E, Arzimanoglou A, et al. (2006) Nocturnal hypermotor seizures, suggesting frontal lobe epilepsy, can originate in the insula. Epilepsia 47: 755-765.

41 Ryvlin P (2006) Avoid falling into the depths of the insular trap. Epileptic Disord 8 Suppl 2: S37-56.

42 Ryvlin P, Kahane P (2005) The hidden causes of surgery-resistant temporal lobe epilepsy: extratemporal or temporal plus? Curr Opin Neurol 18: 125-127.

43 Yasargil MG (1988) Microneurosurgery. Vol. IIIB. Thieme Medical Publishers. New York.

44 YaÅŸargil MG, von Ammon K, Cavazos E, Doczi T, Reeves JD, et al. (1992) Tumours of the limbic and paralimbic systems. Acta Neurochir (Wien) 118: 40-52.

45 Yasargil MG (1996) Microneurosurgery. Vol. IVB. Thieme Medical Publishers. New York.

46 Bertalanffy H, Gilsbach JM, Eggert HR, Seeger W (1991) Microsurgery of deep-seated cavernous angiomas: report of 26 cases. Acta Neurochir (Wien) 108: 91-99.

47 Zentner J, Meyer B, Stangl A, Schramm J (1996) Intrinsic tumors of the insula: a prospective surgical study of 30 patients. J Neurosurg 85: $263-271$

48 Vanaclocha V, Sáiz-Sapena N, García-Casasola C (1997) Surgical treatment of insular gliomas. Acta Neurochir (Wien) 139: 1126-1134.

49 Heffez DS (1997) Stereotactic transsylvian, transinsular approach for deep-seated lesions. Surg Neurol 48: 113-124.

50 Meyer FB, Bates LM, Goerss SJ, Friedman JA, Windschitl WL, et al. (2001) Awake craniotomy for aggressive resection of primary gliomas located in eloquent brain. Mayo Clin Proc 76: 677-687.

51 Wang F, Xu YL, Li ZZ, Liu Z, Sun T (2012) Surgical strategies and seizure outcomes of patients with epilepsy due to insular lesions. Chin J Neurosurg 28: 392-395.

52 Liu Z, Wang F, Xia HC, Qi JH, Song ZM, et al. (2011) Kindling epilepsy models with kainic acid in different parts of rat brain. Chin J Exp Surg 28: 1582-1584.

53 Liu QZ, Wang F, Mou QC, Zhang PS, Xu WZ, et al. (2009) Correlation between rat insular and amygdala electrical kindling epilepsy, J Ningxia Med Univ 25: 339-342.

54 Wang L, Zhang MZ, Zhao JZ, Meng GL, Han XD (2004) Clinical features and minimally invasive surgery of insular lesions: report of 42 cases. Chin Med J (Engl) 117: 1104-1108.

55 Liu QZ, Wang F, Mu QC, Zhang PS, Sun T (2010) [Expressions of GAP4, P38 mRNA and protein in insular electrical kindled rats and its significance]. Zhonghua Yi Xue Za Zhi 90: 1348-1352.

56 Kriegel MF, Roberts DW, Jobst BC (2012) Orbitofrontal and insular epilepsy. J Clin Neurophysiol 29: 385-391.

57 Wang EJ, Wang F, Song ZM, Xu J, Miao L, et al. (2012) [Expression of $\hat{\mathrm{I}}^{\prime}$ subunit of $\hat{\mathrm{I}}^{3}$-aminobutyric acid $\mathrm{A}$ in hippocampus induced by insular-kindled rats]. Zhonghua Yi Xue Za Zhi 92: 1215-1218.

58 Xu WZ, Wang F, Zhang PS, Ren SL, Qi JH, et al. (2011) The expression of BDNF and TrkB in rat hippocampus and the relationship with learning-remembering ability in chronic electronic ignition of insular lobe. J Ningxia Med Univ 33: 304-307.

59 Malak R, Bouthillier A, Carmant L, Cossette P, Giard N, et al. (2009) Microsurgery of epileptic foci in the insular region. J Neurosurg 110: 1153-1163.

60 Lang FF, Olansen NE, DeMonte F, Gokaslan ZL, Holland EC, et al. (2001) Surgical resection of intrinsic insular tumors: complication avoidance. J Neurosurg 95: 638-650.

61 Chang LJ, Yarkoni T, Khaw MW (2013) Decoding the Role of the Insula in Human Cognition: Functional Parcellation and Large-Scale Reverse Inference. Cerebral Cortex March 23: 739-749.

62 Kurth F, Zilles K, Fox PT (2010) A link between the systems: functional differentiation and integration within the human insula revealed by meta-analysis. Brain Struct Funct 214:519-534.

63 Beissner F, Meissner K, Bar KJ (2013) The autonomic brain: an activation likelihood estimation meta- analysis for central processing of autonomic function. The Journal of Neuroscience 33: 10503-10511.

64 Sun T (2014) Insular cortex epilepsy. Chinese Journal of Neurosurgery 30: $976-978$ 